\title{
Diagram Notations for Mobile Work Processes
}

\author{
Sundar Gopalakrishnan and Guttorm Sindre \\ Department of Computer and Information Science, \\ Norwegian University of Science and Technology (NTNU), 7491 Trondheim, Norway \\ \{sundar, guttors\} @idi.ntnu.no
}

\begin{abstract}
In mobile and multi-channel information system, the geographical location and context of the user when performing some task may be important for the design of IT applications. Yet, mainstream process models seldom capture the "where" aspect, such as the location for performing some activity. In previous papers we have performed an initial analytical evaluation and two controlled experiments comparing some notation alternatives. For all these notation alternatives the underlying assumption has been that they should be achieved as fairly small adaptations of existing process notations, using UML Activity Diagrams as an example. In this paper we provide a more comprehensive analysis of the problems, using 9 principles for visual notations proposed by Moody, and considering clean-sheet design of a process notation in addition to minor adaptations of existing ones. The paper demonstrates how this would result in quite different notations, each with their pros and cons.
\end{abstract}

Keywords: Mobile, multi-channel, information system, process model, diagram notation, visual communication.

\section{Introduction}

Business process modelling languages tend not to capture the location of the activities performed. For instance, BPMN [1] and UML activity diagrams [2] capture what (objects), how (sequence and parallelism of activities and decisions), who (swimlanes), when (time triggers and time events), and to a limited extent why (e.g., how a decomposed activity diagram satisfies a higher level activity). For the latter some extensions with process goals have also been suggested [3] - but not the location of the activities performed. For traditional office information systems, where work is performed using desktop computers, it is understandable that physical location has not been given much attention. It is much more important whether a task is performed by the Purchases or Salary department than whether the worker is sitting in office 221 or 325 , and even in a distributed IS setting, it might not be so important if one of the offices is in another country either, since anyway the usage context is quite similar, thus implying similar requirements for the desktop application.

However, as emphasized in [4], the location and context of activities performed is of much higher importance in mobile and multi-channel information systems. For instance, whether a certain information processing task should be performed in the 
office before going out on a power line repair job, in the car while driving, in the terrain while walking and searching for the exact site of damage, while climbing in the power mast to fix the damage, while driving back from the site, or after having returned to the office - could have a large impact both on quality, efficiency, and job satisfaction, and would therefore be an important process design decision. In turn, this decision should also have a lot of impact on what ICT tools would have to be developed to support the work process, and what requirements that these tools would have to satisfy. For instance, if the task were to be performed on foot in a dense forest, this would require different equipment and imply other usability challenges than what a desktop application is normally faced with.

It is therefore interesting to consider specialized process notations that do show location and or context of the worker. This can be done either by small adaptations of existing notations like BPMN or UML activity diagrams, or by a more radical "clean sheet" design of a novel notation specifically supporting the modelling of mobile work processes. In some previous papers [5-7] we have mainly looked at the former alternative, investigating some minor adaptations of UML activity diagrams. The purpose of this paper is to make a more general evaluation of the relative merits of both alternatives, i.e. also opening for proposals of notations that are radically different from the mainstream, using Moody's 9 principles for visual notations [5]. The research questions for this paper are as follows:

RQ1: What are the most plausible visual variables to use for showing location in process models?

RQ2: What are the relative merits of designing from scratch a novel process notation for mobile information systems versus minor adaptations of existing notations?

The rest of the paper is structured as follows: Section 2 presents background and related work in the area, section 3 presents the notation alternatives. Section 4 provides an evaluation of the notation alternatives, and section 5 concludes the paper.

\section{Background and Related Work}

When considering the modelling of location, it is important to differentiate between space and place. The best background for conceptualization of these aspects comes from fields of cartography and CSCW, the latter distinguishing between space and place [8-9]. "Space" describes geometrical arrangements that might structure, constrain, and enable certain forms of movement and interaction; "place" denotes the ways in which settings acquire recognizable and persistent social meaning in the course of interaction. In this work we look at modelling of 'place'. Work combing modelling of space (e.g. in maps) and conceptual aspects are described in [10].

In previous papers we first performed a preliminary analytical evaluation of some notation alternatives in [5], the evaluation based on a semiotic framework [11]. Then we compared the most promising notations in experiments [6-7], which revealed that a notation using coloured nodes for capturing location fared better than using 
pattern-fills or UML notes. However, there are a lot of other possible notations not evaluated yet, especially radically new notations, as the previously mentioned studies focussed on minor adaptations of UML activity diagrams.

Moody [12] defines 9 principles or guidelines for effective visual notations. 1) semiotic clarity means that there should be a 1:1 mapping between graphical symbols and concepts. 2) perceptual discriminability: How easily and accurately can the various graphical symbols be differentiated from each other? It will be easier for the user to distinguish between shapes that are obviously different from a quick glimpse (e.g., squares, circles, triangles) than between shapes that are different only in subtle details (e.g., rectangles with a varying height/width ratio, with or without rounded corners, or with differences in textual font or style inside the rectangle). 3) semantic transparency: How well does a symbol intuitively reflect its meaning? According to Moody, symbols can be either immediate, having a nice intuitive relationship with their corresponding concepts, opaque, having only an abstract relationship, or even worse, they might be perverse, its intuitive interpretation being misleading vs. the represented concept. 4) complexity management: What constructs does the diagram notation have for supporting different levels of abstraction, information filtering, etc.? 5) cognitive integration: Does the notation provide explicit mechanisms to support navigation between different diagrams? 6) visual expressiveness: To what extent does the notation utilize the full range of visual variables available? 7) dual coding: Using text to complement graphics. 8) graphic economy: Avoiding a too large number of different symbols, which would make the notation hard to learn and understand. 9) cognitive fit: Trying to adapt the notation to the audience, i.e. possibly using different dialects with different stakeholder groups. The 9 principles of Moody have already been used in [13] for evaluating the notations of UML, and in [14] for evaluating i*. While those papers focused on evaluating existing modelling notations, our focus is to use the 9 principles to guide proposals for new notations.

There have been several efforts presenting adaptations of diagram notations. Mendling et al. [15] propose to insert small icons inside each business process activity, but not to indicate location, rather the nature of the activity, where the authors identify 25 generic labels. Some examples of concepts represented using such iconic labels are "assess" (using a weight scales icon), "complete" (using a green filled circle with a white check mark), "decide" (using a question mark), "promise" (using a handshake symbol), and "search" (using a magnifying glass symbol) [15] (p.52).

Most closely related to this paper is work by Baumeister et al. [16], where the authors propose some extensions to UML activity diagrams specifically targeting the modelling of mobile systems. In particular, it distinguishes between what the authors call a responsibility centered notation, where swim-lanes are used to indicate "who" performs and action, and a location centered notation, where swim-lanes indicate "where" an action is performed. In the responsibility centered notation location is instead indicated by the addition of textual labels in action or actor nodes, and similarly, the responsible actor is indicated by textually labelled actor objects in the location centered notation. Since responsibility centered notation is the standard usage of swim-lanes, it is location centered notation which represents the major 
novelty or deviation from normal practice. This resembles a notation which was briefly tried but dismissed in [5] because the need for showing responsibility by other means created a lot of extra nodes and edges in the diagram, thus creating a messy diagram that would be hard to comprehend especially for stakeholders with limited technical skills.

Decker [17] also proposes an adapted notation of UML activity diagrams, specifically targeting access control for mobile workflows. Each activity node can be linked to one or a series of location nodes, shown by parallelograms, to indicate that it is compulsory or prohibited to perform certain activities in certain locations. [18] makes a similar proposal for BPMN diagrams, again showing locations by icons with a parallelogram shape. Thus creating separate nodes for locations, this slightly resembles the usage of UML notes to indicate locations, as investigated analytically in [5] and experimentally in [6], however with poorer user performance than an alternative approach of coloured nodes.

Walderhaug et al. [4] used UML notations extensively in the MPOWER project with homecare services and conclude that UML profiles can be used as a mechanism for tool chains based on OMG's Model Driven Architecture (MDA) and UML standards. Work on mobile ontologies by Veijalainen [19] supports the idea of the 'where' aspect as essential in mobile processes, but excludes the 'what' aspect. Larsson [20] proposes the three building blocks for knowing the processes list How, What and Why, adds Who for use oriented design approach but omitted the 'Where' concept.

\section{$3 \quad$ Notation Alternatives}

There are a number of notation alternatives that have so far not been explored in our experiments [6-7], nor by the initial analytical evaluation in [5]. Bertin [21] presents 8 different visual variables that might convey meaning in diagrams:

- planar variables: horizontal position, vertical position

- retinal variables: size, brightness (="value"), texture, colour, orientation, and shape.

As Bertin points out these can be used alone or in combination to give different meanings to diagrammatic constructs, and so far we have only explored the usage of some of these variables. Using planar variables to indicate location of activities would mean that an activity node's placement in the diagram would denote the location where the activity takes place in the real world. Instead using retinal variables, variations in the visual appearance of the activity node would indicate the place where the activity is performed. Figure 1 illustrates a spectrum of possibilities. The first row indicates the planar alternative (embedding activity nodes in pools, in a practical example there would of course be several pools and several activities in each pool), the remaining rows showing retinal alternatives, either just adding text (first row), adding an extra location node (next three rows), or providing variations of the activity node itself, e.g., in the bordering line (last four rows), fill (three rows above those), shape, size, or orientation. Many of these alternatives would not provide good notations, but are included just for completeness. 


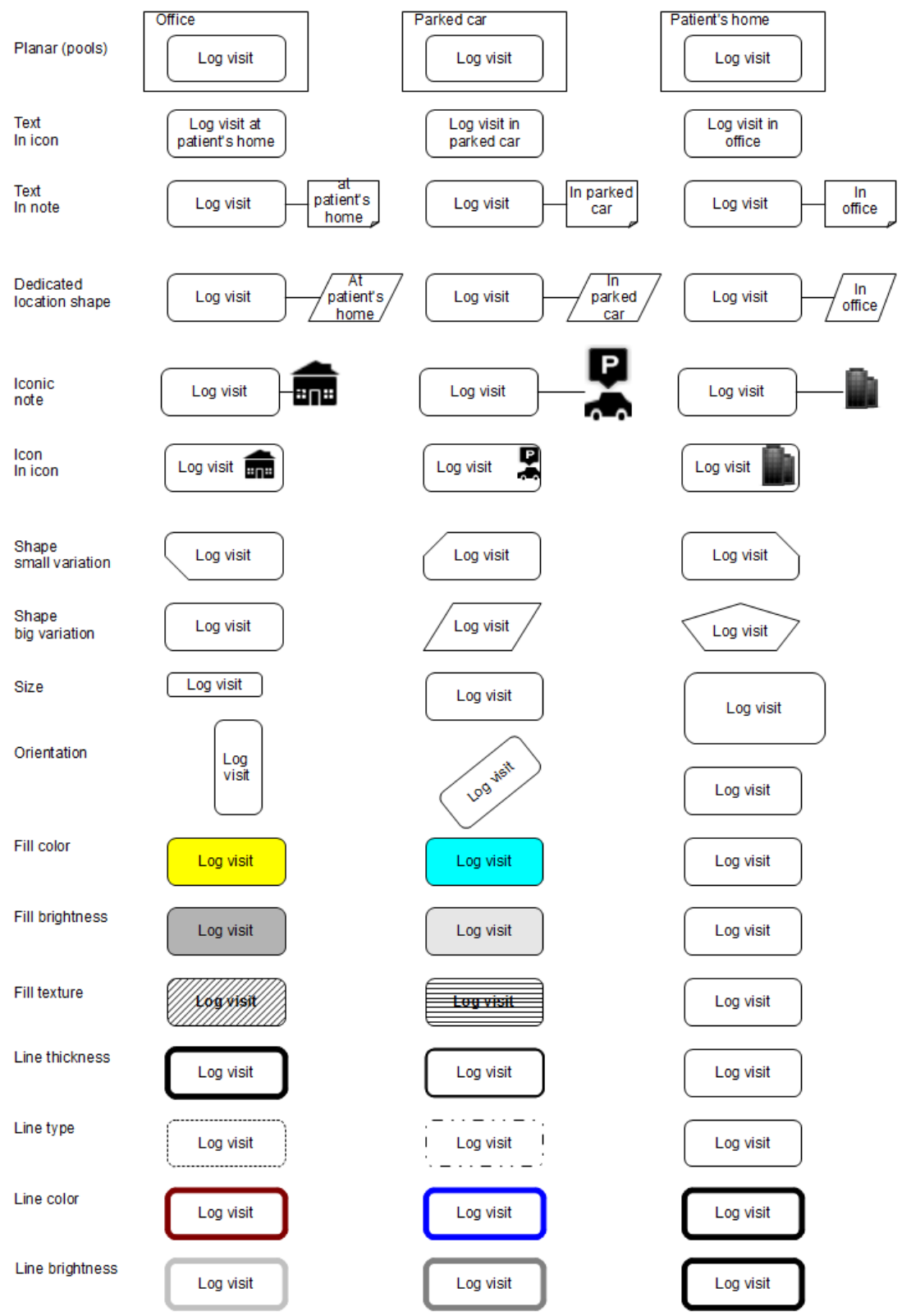

Fig. 1. Showing location by planar (top row) and retinal variables (remaining rows). Fill-colors yellow, blue, white, line colors red, blue, green (if $b / w$ printing).

The first question is whether we should go for an entirely new notation, or a smaller adaptation of existing notation. There are of course pros and cons to both these alternatives: 
- entirely new notation has the advantage that one is free to make optimal choices vs. notation design, specifically fitting the representational challenge at hand. On the other hand, more work is needed to establish the notation and supporting tools, making it less likely that the notation will be adopted by anybody.

- smaller adaptation of existing notation(s) have the advantage of leveraging the investment found in existing tools and analyst knowledge (i.e., more likely to pick up a notation which is a small adaptation of something they already know). On the other hand, there is less freedom in developing the notation since most of the choices made in existing notation must be retained, thus the notation might not be visually optimal.

In the following we will discuss these two possibilities separately, considering entirely new notations in section 3.1 and adaptations in section 3.2.

\subsection{Entirely New Notations}

For an entirely new notation, the usage of planar variables (horizontal and vertical placement) could be the most intuitive choice to indicate geographical location, as this the normal approach for spatial relationships in maps, which most people have somehow been accustomed to without any IS education. As discussed in [10], due to the different meta-meta model of maps and conceptual models, there are differences in how to exploit the nine principles of Moody. Cartography revolves, generally, around geographical information which is strongly reflected in the visualization used. Generally the visualization can be said to comprise three graphic primitives; point, line, and area, and relations between these. This is inherently different from metameta models in conceptual modelling which usually comprise only nodes and links between nodes, in addition to containment. From [10] we have the following guidelines based on the work on MAPQUAL:

1. Clearly discriminate between geographical oriented lines and conceptual lines (relationships)

2. Clearly differentiate between nodes (concept) which are often depicted by a geometric shape, and geographic areas (by texture or colour for instance)

3. Indicate topological information by positioning of conceptual nodes according to the topology where relevant.

4. Position concepts according to their temporal nearness.

5. Use visual variables where appropriate, especially the use of colour and shading for differentiation is necessary for integrated models.

6. Design the visualization based on the participants' cognitive metaphor of the most important information attribute. For instance, temporal attributes tend to be lean towards a sequential metaphor. Spatial attributes, like nearness, tend to lean towards a distance metaphor (i.e. closer is nearer). 
The work in [22] illustrates that the new conceptualization might be promising, in particular when combining space and conceptual aspects. Looking on the different archetypical work-places as discussed in the start of section 2 (stationary in office, stationary visiting, travelling by own means, being transported, wandering) one often needs to differentiate between a limited number of places.

The idea of using swim-lanes for location was briefly tried out in [5] but dismissed because it created messy diagrams. If using swim-lanes pools for location, the challenge becomes how then to show which role or organizational unit is responsible for various tasks. The attempt in [5] was to use the stick-man figure, since this is anyway a well-known symbol in UML. The problem was that links then had to be drawn from every activity to some stick-man figure, and in anything beyond extremely simple process models, crossing links made the diagrams hard to read. However, considering entirely new notation, one does not have to be loyal to UML notation and use the stick man figure, instead the actors for activities can be indicated by means which do not create extra nodes or links in the diagrams, for instance:

- colour or texture. These do not have any obvious intuitive correspondence with various organizational units, thus needing a legend associated to each diagram, although one could imagine developing domain-specific conventions for various organizational units typically involved.

- iconic labels inserted in the activity nodes. These have an advantage over colour in being easier to understand intuitively, for instance HR dept $=$ stick figure, sales $=$ dollar, salaries $=$ pay sack, production $=$ gear wheel, $R \& D=$ question mark, top management = exclamation mark, etc. A downside versus colour might be if presenting huge models with limited space, when icons inside activity nodes might become small and make the diagrams appear overloaded.

Figure 2 and Figure 3 show the same simplified example of a mobile work process in municipal home care, initiated by a shift leader distributing patients on various home care assistants (e.g., depending on patient needs and available assistants, feasible geographical allocation, etc.). Each home care assistant then decides on the visiting sequence of the allocated patients and drives off on the visiting round. On the way to each patient the assistant gets preliminary information about the patient, preferably by audio to allow for sufficient attention to the driving. For each visit, the assistant takes care of the necessary task for the patient and, when returning to the parked car, immediately logs the visit, possibly also registering special needs that must be taken care of on the next visit (e.g., buying new light bulbs). In case the patient's health condition does not appear to be normal, the assistant may contact a nurse who will take further action if necessary.

In Figure 2 swim-lanes are used for denoting the different locations where this work-process is played out, while white and grey-tones for the activity nodes show the actors. Colour would be even more illustrative, but grey-tones were used here for the purpose of $b / w$ printing. In Figure 3 bounding boxes similar to swim-lanes are again used for location, but this time the different locations are even more emphasized by using different background colour inside the bounding boxes, plus some 
illustrative icons (office building, driving car, parked car, homes, and red cross for the clinic) to indicate the nature of the various locations. Instead of using different greytones inside the activity nodes, icons are used also here, to distinguish between activities performed by the shift leader, the home care assistant, and the nurse.

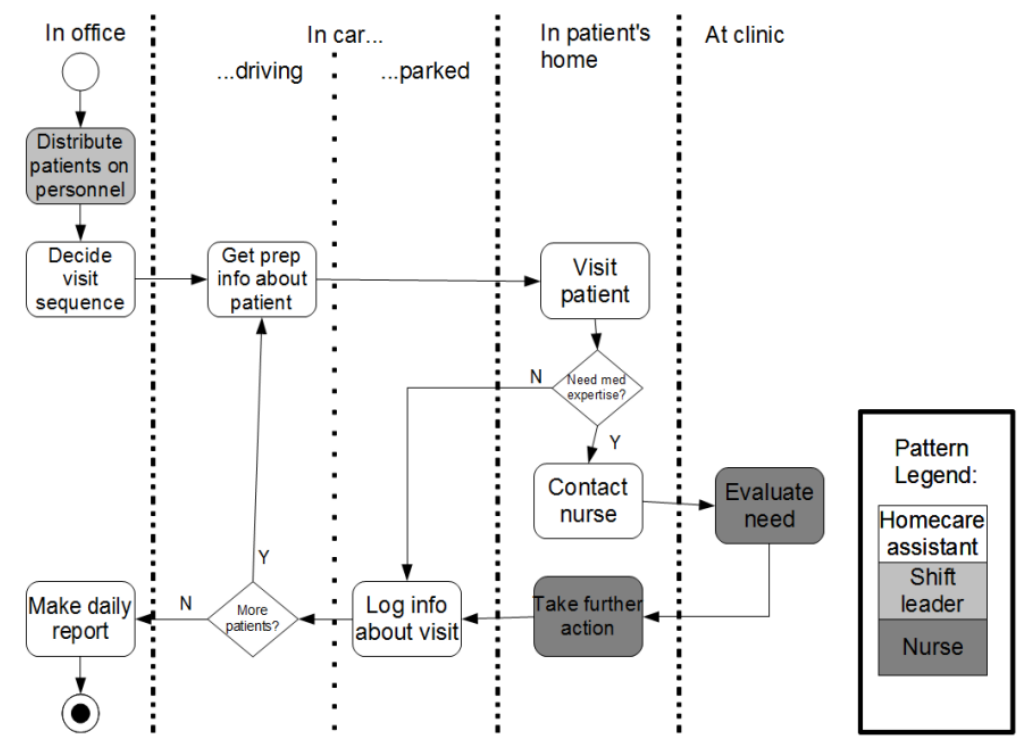

Fig. 2. Distinguishing actors by brightness (grey-tones), swim-lanes for location

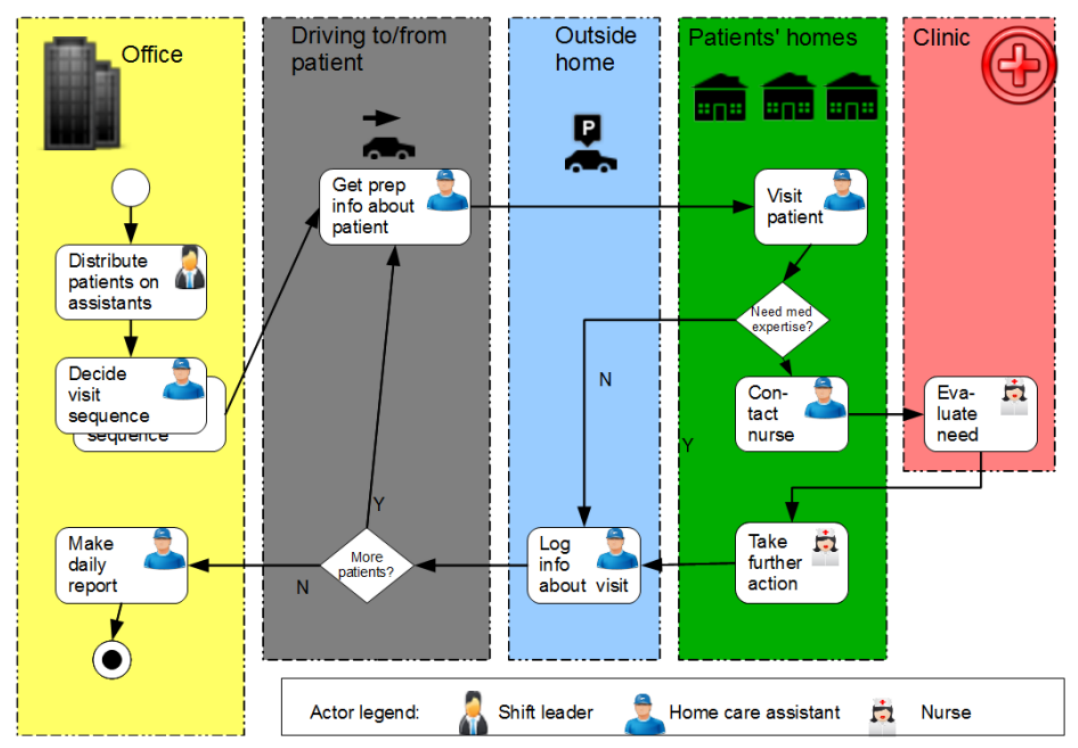

Fig. 3. Distinguishing actors by people icons, pools for location 


\subsection{Adapted Notations}

Existing process modelling approaches like BPMN or UML Activity Diagrams tend to use the planar variables for activity order and possibly organizational placement in swim-lanes or pools. If the goal is to adapt an existing notation, it would make sense to keep using the planar variables for these purposes, so that location would instead have to be shown by retinal variables, cf. Figure 1. Many of these can be dismissed quite quickly using one or more of Moody's 9 principles. For instance, the four lower rows which use variations on the bordering line of the activity node will all be very subtle, with poor perceptual discriminability. Using size will be confusing, as this will intuitively be understood to indicate complexity or importance, not location, giving poor semantic transparency. The same would apply to orientation, intuitively indicating increase or decrease. Using totally different symbols ("shape, big variation") would be a radical change from standard notation and the user would probably be confused that such quite different symbols would all represent activities. On the other hand, the alternative "shape, small variation" suffers from poor perceptual discriminability, and worse the more locations must be distinguished. The alternatives with separate nodes have problems with regards to the principle of complexity management, since they cause a substantial increase in the number of nodes in the diagram, and thus also to the number of links between nodes, easily causing crossing lines and reduced readability. This was probably a key reason why a notation using colour performed significantly better than a baseline notation using UML notes in an experiment [6]. The advantage of using UML notes, on the other hand, is that it does not require any changes to standard UML notation.

In another experiment [7] the alternatives with colour-fills and pattern-fills performed equally well, although opinion-wise the experiment subjects had a strong preference for the colour version. Another possibility that might be tempting to try out is the "icon in icon" alternative. This shares the advantage of colour- and pattern-fills that the number of nodes and links in the diagram is not increased. If one is able to make intuitive icons, it would have an additional advantage in terms of higher semantic transparency. On the other hand, it would have a potential disadvantage for the principle of graphical economy (adding more different symbols to the notation), and in a large model with many small activity nodes, icons may become smallish and hard to read.

In Figure 4 the same home care example as in Figures 2 and 3 is shown again, now using planar variables for responsibility (swim-lanes) and activity order (top to bottom), and instead showing location by pattern fills. Colour got even better results than pattern-fills in the experimental evaluation, but pattern-fills are used as an example here for the purpose of black/white printing. Anyway, whether using patterns, colour or grey-tones, the diagrams would be structurally the same.

Figure 5 then shows the same example instead using icons to indicate the locations of actions. Four different icons are used, an office building for tasks performed in the office, a car with an arrow on top for tasks performed while driving, the same car with a P-sign above for tasks performed while parked, and a house for the patient's home. A legend might be useful for these also, but the much more intuitive relationship between these symbols and what they stand for, means that the user would likely learn and remember them more easily. 


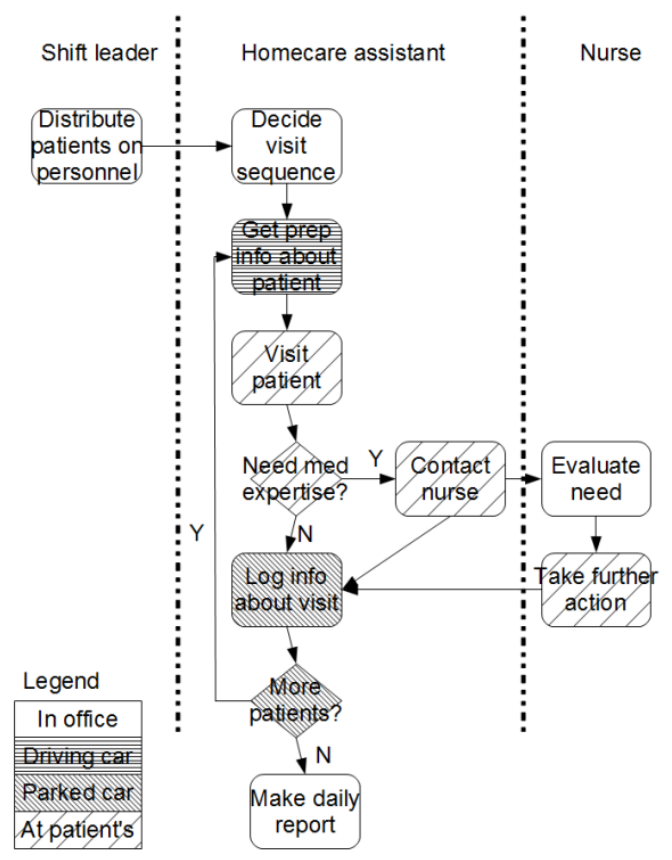

Fig. 4. Home Care Example, using pattern-fills for location

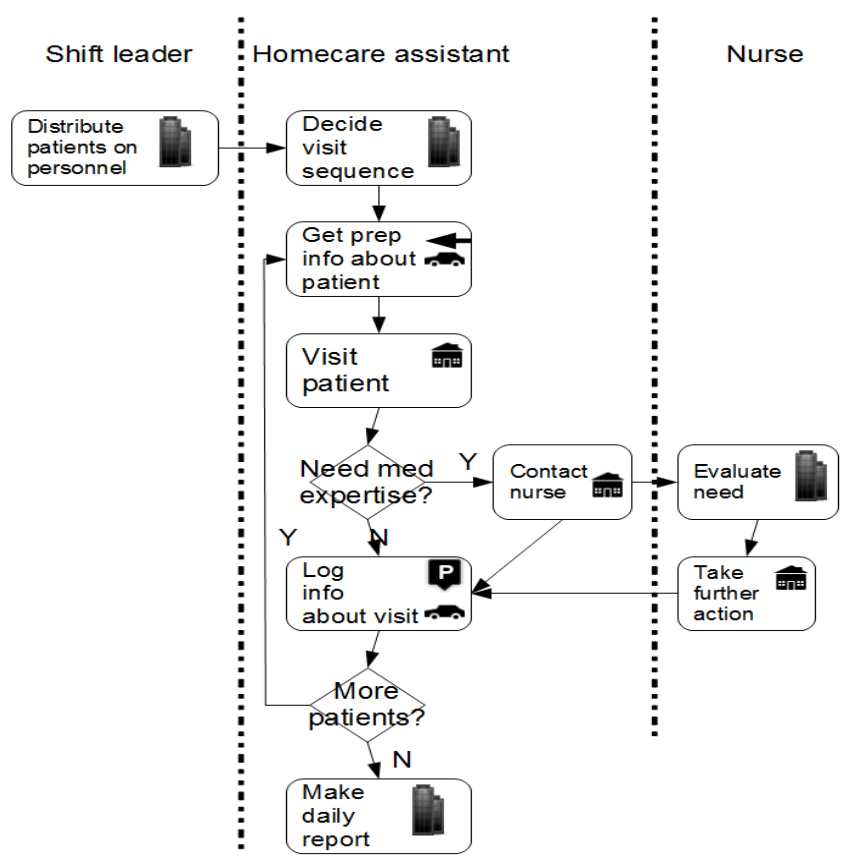

Fig. 5. Showing locations of actions by place icons inside activity nodes 


\section{$4 \quad$ Evaluation of Notations}

Not all of Moody's 9 principles [12] are equally relevant to us. Two of the principles are excluded, namely cognitive integration, because it concerns relationships between several diagrams or diagram types while we are only considering activity diagrams at this point, dual coding, because this concerns the combination of text and visual means - which can be done fairly independent of what visual trick we use for location, and cognitive fit, which concerns the usage of different dialects for different stakeholder groups (where, if anything, we would need representatives from these various groups to perform the evaluations rather than doing it ourselves). This leaves us with 6 of Moody's principles, on the other hand we add one additional criterion which is not in Moody's list, namely support for multiple locations. In our examples in Figure 1, each activity is shows as taking place in one particular location. However, for a mobile information system some activities must be supported in many different locations, so that it is up to the user's preference where to perform it (e.g., either in the office or in the car). Some activities might even take place in several places at once, for instance if performed by two collaborating persons in different locations. The need to attach several locations to some activities might obviously cause additional challenges to our notation alternatives.

Table 1. Evaluation of notations, responsibility-centered alternatives above grey line, locationcentered alternatives below

\begin{tabular}{|l|c|c|c|c|c|c|c|c|}
\hline Show location by... & SC & PD & ST & CM & VE & GE & ML & Sum \\
\hline Text in activity node & - & - & & & -- & ++ & & -2 \\
\hline Text in note & - & - & & -- & -- & ++ & + & -3 \\
\hline Dedicated location shape & & & & -- & -- & - & ++ & -3 \\
\hline Iconic note & + & ++ & ++ & - & - & -- & ++ & +3 \\
\hline Icon in icon & + & ++ & ++ & + & - & -- & & +3 \\
\hline Shape, small variation & + & - & & + & -- & & - & -2 \\
\hline Shape, big variation & + & + & & + & -- & -- & -- & -3 \\
\hline Fill colour & + & ++ & - & + & + & + & & +5 \\
\hline Fill brightness & + & + & - & + & + & + & - & +3 \\
\hline Fill texture & + & + & & + & + & + & - & +4 \\
\hline Planar, resposibility by & & & & & & & & \\
\hline Iconic note & + & ++ & ++ & - & - & -- & -- & -1 \\
\hline Icon in icon & + & ++ & ++ & + & - & -- & -- & +1 \\
\hline Fill colour & + & ++ & & + & + & + & -- & +4 \\
\hline Fill brightness & + & + & & + & + & + & -- & +3 \\
\hline Fill texture & + & + & + & + & + & + & -- & +4 \\
\hline
\end{tabular}

The rows in Table 1 are various notation alternatives for location, for space reasons we omitted some that were obviously poor already in the discussion of Figure 1. The columns are the criteria used $(\mathrm{SC}=$ semiotic clarity, $\mathrm{PD}=$ perceptual discriminability, $\mathrm{ST}=$ semantic transparency, $\mathrm{CM}=$ complexity management, $\mathrm{VE}=$ visual expressiveness, GE = graphical economy, and $\mathrm{ML}=$ multiple locations. The scores range from - - (very poor), through poor, neutral, good, to very good (++). The tows 
above the grey line are for notation with small adaptation, i.e., planar variables used for responsibility so location must use retinal variables. Below the grey lines are alternatives using planar variables for location, so that responsibility must be shown by retinal variables. Here we only included the 5 best alternatives from the upper half, since the relative merits of the various alternatives will be pretty much the same whether the retinal variables are supposed to depict location or responsibility.

For space reasons we cannot explain all the 105 marks in this table in detail, more information can be found in a technical report [23]. The analytical evaluation was performed by both authors independently, then going through a consensus process afterwards, to reduce the threat of scoring error and arbitrary interpretation of evaluation criteria. However, both evaluators - although having different cultural backgrounds - came from the same university, and having a four year scientific relationship (supervisor and phd student), so the results must be taken with caution.

The alternatives using fills (either colour, grey-tones or patterns) generally scored well in the evaluations, because they do not introduce new nodes or links (i.e., good for complexity management), put to use visual variables that are not used for any other purposes in the notation in question (i.e., good for visual expressiveness), and are fairly easy to discriminate visually (i.e., good for perceptual discriminability). This is especially true for colour (except for color-blind users), but brightness and texture will also be fine as long as each diagram has a limit of 4-5 different actors or locations that must be distinguished. Another good alternative is the use of meaningful icons inside the activity nodes ("icon in icon"). These have a big advantage where colour-fills and similar alternatives were not impressive, namely in being intuitively understandable, i.e. good for semantic transparency.

As for the choice between the radical, location centered notations using planar variables for location (lower part of Table 1), or the less radical responsibility centered notations using retinal variables for location (upper part), the planar alternatives might be slightly better for semantic transparency, but have problems with multiple locations, in which case activity nodes might have to be duplicated in several swim-lanes. For multiple locations, the dedicated location shape or iconic note will be the best, since an activity node can trivially be connected to a number of location nodes. Icon in icon or colour fills are not too bad either, since one can put several icons or colours inside the same node.

\section{Discussion and Conclusion}

Our first research question was about the most plausible visual variables to use for showing location in process models, and the analysis has indicated a two-level choice:

- $\quad$ showing location by planar variables, in which case responsibility must instead be shown by other means; the best seem to be fills in the activity nodes (e.g., colour, grey-tones, patterns) or meaningful icons in the activity nodes.

- Showing responsibility by planar variables, in which case location must instead be shown by retinal variables, the most interesting again seeming to be fills or meaningful icons. 
The second research question was about the relative merits of radical locationcentered notations versus smaller adaptations of mainstream responsibility-centered notations. The choice between these depends on several factors. If similarity to mainstream notations is important, it will be a good idea to keep showing responsibility by planar variables. On the other hand, for semantic transparency, it might be better to use planar variables for location. There is, however, a potential problem with models where some activities can be performed in multiple locations, since it would cause clumsy diagrams if activity nodes must be duplicated in several location pools. For activities supposed to be performable everywhere, there could be a workaround by designating a special "anywhere" pool, but this would not solve the problem for activities that should be supported in a couple of different places, but not all. Hence, while the usage of planar variables for location looks nice as long as the models only need to depict each activity as performed in one place, it might in the more general case not be as interesting as initially believed. From the evaluation tables, the aspect not shown by planar variables (whether this be location or organizational responsibility) can feasibly be indicated for instance by colours or similar fills inside the activity nodes, or by meaningful icons inside the activity nodes ("icon in icon") -- or, if multiple locations for each activity is a major issue, possibly putting the iconic nodes as separate nodes in their own right ("iconic node"). A major point made by Moody as reflected in the principle of cognitive fit [12], however, is that one should not have to make a choice of only one notation here. A reasonably sophisticated modelling tool should easily allow to switch between different visualizations of the same underlying conceptual model - depending on the preference of the user. So, a quick menu choice could determine whether to hide or show locations in a diagram, using a location-centered or responsibility-centered notation, whether to use colour or pattern-fills or icons - or other visual variables.

A major shortcoming of this work so far is that most of the example notations are only evaluated analytically, which does not allow for as certain conclusions as would be possible with empirical data. We have done experiments comparing the responsibility-centered "text in note", "color-fill", and "pattern-fill" notations, showing that color-fill performed better than notes and was more preferable to users than pattern-fills. However, the "icon in icon" alternative has not yet been tried in experiments, as have none of the location-centered alternatives. An obvious step for further work would therefore be to run more experiments, and also using more varied participant groups than have been used so far (CS students). Another interesting continuation would be case-studies with larger industrial examples.

Furthermore, it is not evident that swim-lanes or pools must be used at all. It could also be possible to experiment with notations where planar variables only indicate activity sequencing (e.g., top to bottom) or parallelism (e.g., left-right), showing both location, responsibility and other properties (e.g. time) with combinations of fills, icons, and text. After all, while swim-lanes or pools are visually appealing and have some evident advantages - for instance making it quick for each stakeholder to see what activities he is responsible for if swim-lanes are used for responsibility - they might also increase diagram complexity in many cases. For instance, activities which are subsequent and which would therefore intuitively be close in the diagram, might 
have to be drawn far apart because they belong to different swim-lanes - which creates longer links and potentially also more crossing links. Again, in a good modelling tool it should be possible to switch quickly between a display using swimlanes or pools to one avoiding it - as well as to enhance or suppress any particular aspect of the model, e.g., being able to select whether the model is to show location of the activities or not. The study of tool support for the various diagram styles discussed in this paper must therefore also be a topic for further work.

\section{References}

1. Wohed, P., et al.: On the Suitability of BPMN for Business Process Modelling. In: Dustdar, S., Fiadeiro, J., Sheth, A. (eds.) BPM 2006. LNCS, vol. 4102, pp. 161-176. Springer, Heidelberg (2006)

2. Dumas, M., ter Hofstede, A.: UML Activity Diagrams as a Workflow Specification Language. In: Gogolla, M., Kobryn, C. (eds.) UML 2001. LNCS, vol. 2185, pp. 76-90. Springer, Heidelberg (2001)

3. Korherr, B., List, B.: Extending the UML 2 Activity Diagram with Business Process Goals and Performance Measures and the Mapping to BPEL. In: Roddick, J., Benjamins, V.R., Si-said Cherfi, S., Chiang, R., Claramunt, C., Elmasri, R.A., Grandi, F., Han, H., Hepp, M., Lytras, M.D., Mišić, V.B., Poels, G., Song, I.-Y., Trujillo, J., Vangenot, C. (eds.) ER Workshops 2006. LNCS, vol. 4231, pp. 7-18. Springer, Heidelberg (2006)

4. Walderhaug, S., Stav, E., Mikalsen, M.: Experiences from Model-Driven Development of Homecare Services: UML Profiles and Domain Models. In: Chaudron, M.R.V. (ed.) MODELS 2008. LNCS, vol. 5421, pp. 199-212. Springer, Heidelberg (2009)

5. Gopalakrishnan, S., Sindre, G.: Alternative Process Notations for Mobile Information Systems. In: Popplewell, K., et al. (eds.) Enterprise Interoperability IV. Making the Internet of the Future for the Future of Enterprise, pp. 13-23. Springer, London (2010)

6. Gopalakrishnan, S., Krogstie, J., Sindre, G.: Adapting UML Activity Diagrams for Mobile Work Process Modelling: Experimental Comparison of Two Notation Alternatives. In: van Bommel, P., Hoppenbrouwers, S., Overbeek, S., Proper, E., Barjis, J. (eds.) PoEM 2010. LNBIP, vol. 68, pp. 145-161. Springer, Heidelberg (2010)

7. Gopalakrishnan, S., Krogstie, J., Sindre, G.: Adapted UML Activity Diagrams for Mobile Work Processes: Experimental comparison of colour and pattern fills. In: Halpin, T., Nurcan, S., Krogstie, J., Soffer, P., Proper, E., Schmidt, R., Bider, I. (eds.) BPMDS 2011 and EMMSAD 2011. LNBIP, vol. 81, pp. 314-331. Springer, Heidelberg (2011)

8. Dourish, P.: Re-space-ing place: "place" and "space" ten years on. In: Proceedings of the 2006 20th Anniversary Conference on Computer Supported Cooperative Work, pp. 299 308. ACM, Banff (2006)

9. Harrison, S., Dourish, P.: Re-place-ing space: the roles of place and space in collaborative systems. In: Proceedings of the 1996 ACM Conference on Computer Supported Cooperative Work, pp. 67-76. ACM, Boston (1996)

10. Nossum, A., Krogstie, J.: Integrated Quality of Models and Quality of Maps. In: Halpin, T., Krogstie, J., Nurcan, S., Proper, E., Schmidt, R., Soffer, P., Ukor, R. (eds.) Enterprise, Business-Process and Information Systems Modeling. LNBIP, vol. 29, pp. 264-276. Springer, Heidelberg (2009) 
11. Krogstie, J.: Using a Semiotic Framework to Evaluate UML for the Development of Models of High Quality. In: Siau, K., Halpin, T. (eds.) Unified Modeling Language: Systems Analysis, Design, and Development Issues, pp. 89-106. IDEA Group Publishing, USA (2001)

12. Moody, D.L.: The "Physics" of Notations: Toward a Scientific Basis for Constructing Visual Notations in Software Engineering. IEEE Transactions on Software Engineering 35, 756-779 (2009)

13. Moody, D.L., van Hillegersberg, J.: Evaluating the Visual Syntax of UML: An Analysis of the Cognitive Effectiveness of the UML Family of Diagrams. In: Gašević, D., Lämmel, R., Van Wyk, E. (eds.) SLE 2008. LNCS, vol. 5452, pp. 16-34. Springer, Heidelberg (2009)

14. Moody, D.L.: Improving the Effectiveness of Visual Representations in Requirements Engineering: An Evaluation of $i^{*}$ Visual Syntax. In: Patrick, H., Raimundas, M. (eds.) IEEE International Conference on Requirements Engineering, pp. 171-180. IEEE, Atlanta (2009)

15. Mendling, J., Recker, J., Reijers, H.A.: On the Usage of Labels and Icons in Business Process Models. International Journal of Information System Modeling and Design 1(2), 40-58 (2010)

16. Baumeister, H., et al.: Extending Activity Diagrams to Model Mobile Systems. In: Aksit, M., Mezini, M., Unland, R. (eds.) NODe 2002. LNCS, vol. 2591, pp. 278-293. Springer, Heidelberg (2003)

17. Decker, M.: Modelling Location-Aware Access Control Constraints for Mobile Workflows with UML Activity Diagrams. In: 3rd International Conference on Mobile Ubiquitous Computing, Systems, Services and Technologies, Sliema, Malta (2009)

18. Decker, M., et al.: Modeling Mobile Workflows with BPMN. In: 2010 Ninth International Conference on Mobile Business and 2010 Ninth Global Mobility Roundtable, ICMB-GMR (2010)

19. Veijalainen, J.: Developing Mobile Ontologies; who, why, where, and how? In: International Conference on Mobile Data Management, pp. 398-401. IEEE, Manheim (2007)

20. Larsson, A.V.: Designing for use in a future Context - Five Case Studies in Retrospect. In: Institute of Technology. Linkoping University, Linkoping (2003)

21. Bertin, J.: Semiology of Graphics: Diagrams, Networks, Maps. University of Wisconsin Press, Madison (1983)

22. Nossum, A.: Visual Representation of Integrated Information. Dept. of Computer and Information Science. Norwegian Technical University of Science and Technology Trondheim, Norway (2009)

23. Gopalakrishnan, S., Sindre, G.: Analytical Evaluation of Notational Adaptations to Capture Location of Activities in Process Models, Technical report M3W-1, Norwegian University of Science and Technology (NTNU): Trondheim. (2011), http://www. idi.ntnu.no/ guttors/m3w1.pdf 